\title{
MODELING RELATIVISTIC ELECTRON BEAMS WITH FINITE-ELEMENT RAY-TRACING CODES*
}

\author{
$\underline{\text { S. Humphries, Jr..+ }}{ }^{\# \text {, Field Precision, Albuquerque, NM }}$ \\ J. Petillo, SAIC, Burlington, MA
}

\begin{abstract}
Ray-tracing codes determine self-consistent electric fields in high-current electron devices by an iterative procedure of orbit tracking and space-charge deposition with field recalculation. Methods to find beam-generated magnetic fields for relativistic beams are less standardized. Existing approaches (like ray counting or the relativistic transverse force approximation) have limited accuracy and do not include effects of current flow in source and collector electrodes. We describe a new method for two-dimensional finite-element codes where the beam current is deposited on the faces of the conformal elements of the electrostatic mesh. The resulting balanced calculations of electric and magnetic forces are resistant to numerical filamentation instabilities. With simple rules it is possible to assign boundary currents even in complex structures.
\end{abstract}

\section{CURRENT ASSIGNMENT TO ELEMENT FACES}

Figure 1 shows the conformal mesh used in the ETrak code to represent a high-current electron gun. The solution volume is divided into a number of small volumes (or elements) with triangular cross-section. In low-energy devices, the beam behavior is dominated by electric fields. In this case, the following procedure is used to find particle orbits consistent with applied and space-charge electric fields [1].

- Start with a vacuum solution for electrostatic potential at vertices.

- Initiate several model electrons near an emission surface, each carrying a portion of the beam current $I_{i}$. The model particles can be specified or determined by the code from a local application of the Child law.

- Integrate the orbit of each particle using a time step $\Delta t$, depositing charge $\mathrm{I}_{\mathrm{i}} \Delta \mathrm{t}$ in each element that the particle crosses.

\footnotetext{
"Work supported by Los Alamos National Laboratory and the USDOE under Contract DE-FG04-97AL77993 and Subcontract 4400000474 from Science Applications International Corporation under Contract N00014-97-C-2076 from the Naval Research Laboratory.

${ }^{\#}$ Email: humphries@fieldp.com

Permanent address, Dept. of Electrical and Computer Engineering, University of New Mexico, Albuquerque, NM 87131
}

- Recalculate the fields including the electron space charge.

- Repeat the orbit and field calculations until the solution converges.

Some ray-tracing codes use finite-difference methods on a regular mesh $[2,3,4]$ and others employ finite-element methods [5,6]. The advantage of the finite-element approach with conformal mesh is apparent is Fig. 1. Accurate field calculations are possible on curved surfaces and every part of the solution volume has a unique material identity. In ETrak elements are divided into regions that share common characteristics. For example the tan elements in Fig. 1 are part of the cathode electrode, grey elements comprise the anode, and white elements represent vacuum. For particle tracking element properties are divided into three types: vacuum, material and secondary material. Electrons propagate through vacuum materials and stop when they enter a material element. Secondary elements are discussed in Ref. [7].

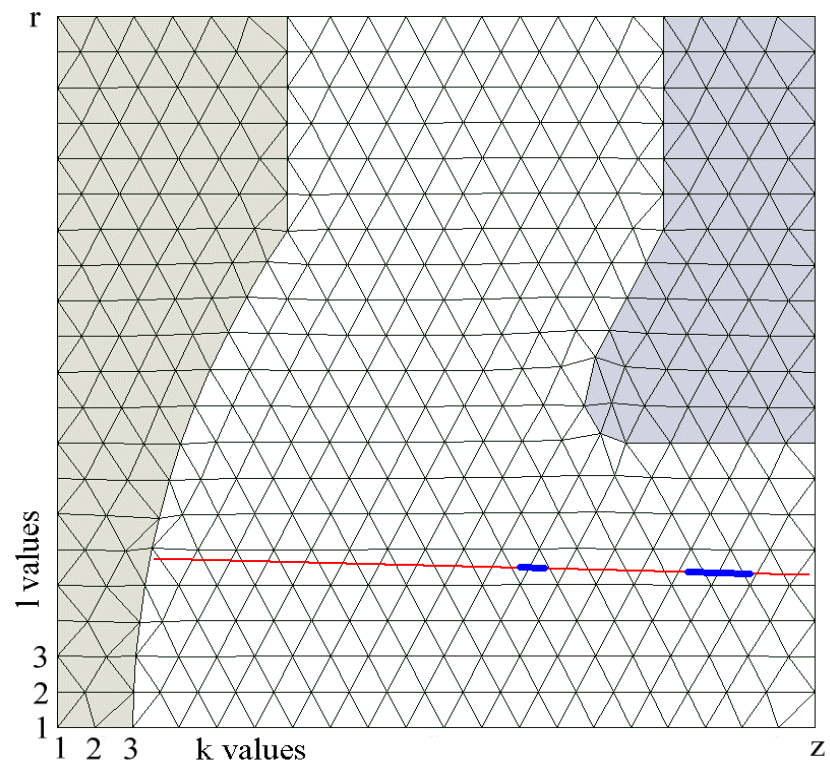

Figure 1: Schematic view of a conformal mesh for a raytracing solution. The colored elements represent material regions. The red line is a model electron trajectory. The marked segments represent the particle orbit vector over a single time step for short and long values of $\Delta \mathrm{t}$. 
Next consider assignment of electron current on the conformal mesh to calculate beam-generated magnetic fields. To satisfy current conservation, the current must be associated with element faces rather than volumes. Electrostatic field interpolations during tracking already provide information on the elements occupied by electrons at times $t$ and $t+\Delta t$. Figure 1 shows two incremental orbit vectors for short and long values of $\Delta \mathrm{t}$. In the first case, the electron crosses to an adjacent element. Here the current $+\mathrm{I}_{\mathrm{i}}$ is assigned to the common face if the particle moves in the positive $\mathrm{z}$-direction, while the value $-I_{i}$ is assigned if the particle moves backward. When ETrak detects that an electron has crossed several elements in a step, the code makes a local search of faces near the average position and assigns $\uplus_{\mathrm{i}}$ to any that intersect the increment particle vector. Face current is always assigned before terminating an electron orbit that enters a material element. This convention ensures correct current assignment to collector surfaces. After tracking all model electrons the process yields a complete set of face currents that satisfy the condition that the divergence of $\mathbf{j}$ is zero.

\section{ENCLOSED CURRENT AND TOROIDAL MAGNETIC FIELD}

After all orbits are tracked, the face current information is used to find values of enclosed current and $\mathrm{B}_{\theta}$, both vertex quantities. The enclosed current is the total beam current that passes between the point and the axis. It is relatively easy to find enclosed current in the structured mesh of ETrak. Following Fig. 1, vertices are labeled in the horizontal direction $(z)$ with the index $k$ and in the vertical direction $(r)$ with index $l$. The procedure for standard vacuum points is first to set the enclosed current equal to zero along the row $l=1$. Next the code works outward toward increasing $l$, processing all vertices in a logical row. ETrak uses a mesh where all standard vertices are surrounded by six elements and connected to six vertices along six faces. Figure 2 shows the face and vertex numbering conventions around Vertex 0 . Faces 4 and 5 connect to vertices with smaller values of $l, 3$ and 6 to vertices on the same tow, and $l$ and 2 to higher $l$.

At each point, the code checks connections to points 4 and 5 on row $1-1$. These points should already have values of enclosed current, $\mathrm{I}_{4}$ and $\mathrm{I}_{5}$. The enclosed current at the point is given by

$$
\begin{aligned}
& \Delta z_{4}=z_{0}-z_{4}, \Delta z_{5}=z_{5}-z_{0}, \\
& \bar{I}=\frac{I_{4} \Delta z_{5}+I_{5} \Delta z_{4}}{\Delta z_{4}+\Delta z_{5}}, \\
& \Delta \bar{I}=\frac{\Delta I_{4} \Delta z_{5}+\Delta I_{5} \Delta z_{4}}{\Delta z_{4}+\Delta z_{5}}, \\
& I_{0}=\bar{I}+\Delta \bar{I} .
\end{aligned}
$$

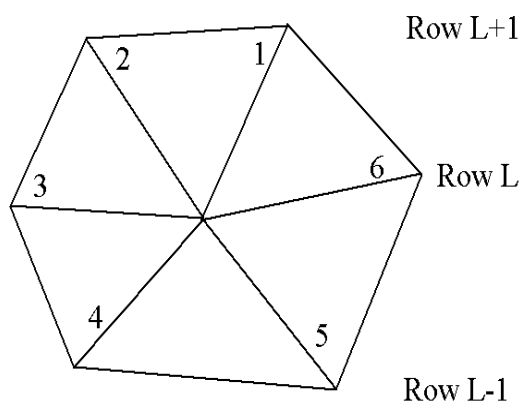

Figure : Relative face and vertex numbers at Point 0 .

The equation involves vertex coordinates $\mathrm{z}_{4}, \mathrm{z}_{5}$ and $\mathrm{z}_{0}$ and connecting face currents $\Delta \mathrm{I}_{4}$ and $\Delta \mathrm{I}_{5}$. Weighting in the formula gives preference to current on vertical faces.

Field averaging between iteration cycles is essential for the stability of the ray-tracing technique. Consider, for example, space-charge assignment for Child law emission. On one cycle, a strong electric field near the cathode would lead to large particle currents. The high space charge would suppress the local field on the next cycle. The solution is to average the newly-calculated space-charge with values from previous cycles. In ETrak the same averaging algorithm is used for beam-generated magnetic field to preserve the balance of forces.

$$
B_{\theta}(\text { new })=\varsigma \frac{\mu_{\text {olenc }}}{2 \pi r}+(1-\varsigma) B_{\theta}(\text { old }) .
$$

In the equation, the quantity $\zeta$ is a number less than unity.

\section{BOUNDARY CONDITIONS}

For valid interpolations of $B_{\theta}$ over the solution volume, material vertices adjacent to vacuum must have correct values of enclosed current. There are two classes of boundary vertices:

- Emission points. These should include the current of the emitted beam even though the electrons do not cross the associated faces.

- Collector vertices. Particles pass through some of the connected faces when stopping in a material. The enclosed current must reflect the incident beam current over all enclosed faces.

Regarding the emission surface, the challenge is to define a method that works for all cathode shapes and arbitrary distributions of electrons. In ETrak an emission surface is a special set of vertices marked as a line region during mesh generation. The program analyzes the mesh, making a list of all faces on the emission surface and confirming that they are adjacent to vacuum. The set of nearby initial electrons is either specified by the user or generated by the code using a space-charge algorithm. In either case the code processes the list of electrons, 
associating each particle with the closest emission face. The present particle currents are assigned to the faces at the beginning of each iteration cycle. To account for concave spherical cathodes and other reentrant structures, the assigned current is always positive, regardless of the emission direction.

The next task is to assign face currents to general material boundaries. For the enclosed current calculation all material regions are treated as perfect conductors. The following procedure is used to process boundary vertices.

- Make a list of all vertices adjacent to material regions and connected to at least one vacuum element.

- Track electron orbits and assign face currents.

- Carry out the enclosed current calculation described in the previous section. Handle boundary points the same as standard points if they have one or more downward connections.

- If a boundary point has no downward connection to a processed vertex, use the following rule. Check Points 3 and 6 . If one of the points has been processed, the enclosed current at Point 0 equals the enclosed current of the neighbor plus the face current of Side 6 or 3.

- If there are no sideways connections, check upward connections to Vertices 1 and 2. In this case, the enclosed current equals the enclosed current of the upper point minus the face current of Side 1 or 2 .

In most situations, the rule propagates the enclosed current over the boundary correctly, even for highly reentrant collector structures.

\section{BENCHMARK CALCULATIONS}

ETrak was applied to a variety of high-voltage gun designs previous treated with Trak 4.0[8]. The code showed good agreement and smooth beam distributions without use of the relativistic force approximation. The solution of Figure 3 demonstrates the accuracy of the technique. The test addresses free expansion of a uniform current-density, $1 \mathrm{MeV}, 617.4 \mathrm{~A}$ electron beam with an initial radius of $1.0 \mathrm{~cm}$. The plot shows computed orbits of 20 model electrons and contours of constant $\mathrm{B}_{\theta}$. Here the transverse forces are created solely by the beam. The electric and magnetic forces balance to within $1 / \gamma^{2}=0.114$. In the absence of the downstream collector the code predicts an envelope expansion over the $50 \mathrm{~cm}$ solution length within 0.3 per cent of the analytic prediction [9]. Figure 3 shows a solution with the addition of a grounded collector with spherical tip (note that there is a $5 X$ vertical exaggeration in the plot). The entrance surface is defined as an emission plane and is treated as a Neumann boundary for the electric field calculation. The parallel magnetic field contours show the effectiveness of the emission surface current assignment. The beam follows the free expansion curve until it reaches the vicinity of the collector. The rod partially cancels the beam electric field allowing the magnetic forces to predominate. In this way, the smalldiameter rod collects the full beam current. Note that this effect depends critically on the correct spatial variation of enclosed current over the outer surface of the collector. The parallel magnetic contours outside the beam confirm that the method conserves current.

\section{REFERENCES}

[1] See, for instance, S. Humphries, Field Solutions on Computers (CRC Press, Boca Raton, 1997), Sect. 10.7.

[2] W.B. Herrmannsfeldt (Stanford Linear Accelerator Center, SLAC331,1988), unpublished.

[3] A.C. Paul (Lawrence Berkeley Laboratory,LBL-13241,1982), unpublished.

[4] J.E. Boers (Sandia National Laboratories, SAND 79-1027,1980), unpublished.

[5] R. True, IEEE Trans. Nucl. Sci. NS-32, 2611 (1985).

[6] S. Humphries, J. Comp. Phys. 125, 488 (1996).

[7] S. Humphries, N. Dionne and J. Petillo, Secondary-electron emission models for charged-particle simulations on a conformal triangular mesh, submitted to Phys Rev Special Topics Accelerators and Beams.

[8] S. Humphries, in R. Ryne (ed) Computational Accelerator Physics (APS, New York, 1994), 597.

[9] See, for instance, S. Humphries, Charged Particle Beams (Wiley, New York, 1990), Sect. 5.4.

Figure 3: Relativistic beam impinging on a grounded collector. Dimensions in $\mathrm{cm}$.

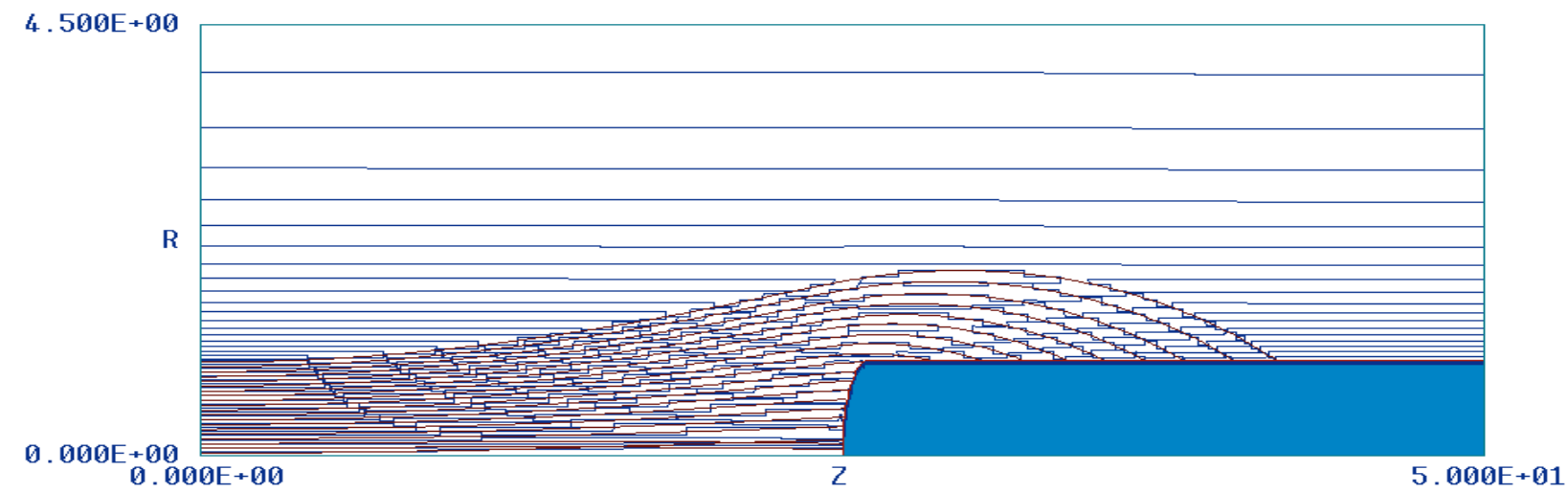

